\title{
EchoGéo
}

$28 \mid 2014$

Police : les espaces de l'ordre, l'ordre en espace

\section{La participation sociale aux activités policières dans les quartiers populaires de Lima}

Entre coproduction du contrôle territorial et reproduction des inégalités socio-spatiales

\section{Camille Boutron}

\section{(2) OpenEdition}

\section{Journals}

Édition électronique

URL : https://journals.openedition.org/echogeo/13869

DOI : $10.4000 /$ echogeo.13869

ISSN : 1963-1197

Éditeur

Pôle de recherche pour l'organisation et la diffusion de l'information géographique (CNRS UMR 8586)

Référence électronique

Camille Boutron, « La participation sociale aux activités policières dans les quartiers populaires de Lima », EchoGéo [En ligne], 28 | 2014, mis en ligne le 08 juillet 2014, consulté le 31 juillet 2021. URL : http://journals.openedition.org/echogeo/13869; DOI : https://doi.org/10.4000/echogeo.13869

Ce document a été généré automatiquement le 31 juillet 2021.

EchoGéo est mis à disposition selon les termes de la licence Creative Commons Attribution - Pas d'Utilisation Commerciale - Pas de Modification 4.0 International (CC BY-NC-ND) 


\section{La participation sociale aux activités policières dans les quartiers populaires de Lima}

Entre coproduction du contrôle territorial et reproduction des inégalités socio-spatiales

\section{Camille Boutron}

\section{Introduction : évolution du concept de " sécurité » et des politiques publiques de maintien de l'ordre en Amérique latine}

1 Alors que la fin de la Guerre Froide a permis un renouvellement du concept de sécurité au niveau international, qui tend alors à se détacher de la vision «Étato-centrique du monde » jusque-là prédominante (Gervais, Roussel, 1998), les transitions démocratiques que l'on peut observer en Amérique latine à la même époque ont eu pour conséquence l'abandon de la doctrine de sécurité nationale au profit d'un nouveau concept de sécurité collective dite citoyenne. Si la doctrine de sécurité nationale veillait théoriquement à protéger avant tout l'intégrité des États plutôt que celle des individus, la sécurité citoyenne est présentée quant à elle comme un instrument de garantie de la démocratie (Davila Altamirano, 2011). Elle désigne ainsi l'ensemble des mesures et initiatives politiques concrètes visant à éradiquer toute chose pouvant porter atteinte à la sécurité collective, dès lors conçue comme un droit individuel et une expression du développement.

2 Les perceptions sociales de l'insécurité sont devenues des enjeux politiques majeurs et le maintien de l'ordre public représente l'une des principales préoccupations des latino-américains (Lagos, Dammert, 2012, p. 5). Dans un tel contexte, les politiques de sécurité publique ont ainsi plutôt tendance à s'appuyer encore principalement sur les acteurs traditionnels du maintien de l'ordre, qui repose lui-même essentiellement sur les institutions policières. Or il est aujourd'hui possible de dire que la grande majorité 
des États latino-américains ne sont pas capables d'assumer à eux-seuls le maintien de l'ordre public, alors qu'un grand nombre d'entre eux apparaissent encore comme les héritiers d'un passé violent ayant laissé son empreinte au sein des représentations et pratiques du quotidien, laissant subsister une méfiance de la population vis-à-vis des institutions publiques dotées de la force coercitive. Principales représentantes des forces de l'ordre, les institutions policières sont ainsi loin de faire l'unanimité, et dans les grandes métropoles la fracture entre les institutions policières et la société civile reste particulièrement visible. Ainsi à Lima, capitale péruvienne, près de $70 \%$ des liméniens n'ont pas confiance dans leur police nationale (Basombrio, 2007, p. 47). Parmi les raisons invoquées pour expliquer ce rejet figurent le manque de moyens accordés à la police, la radicalisation et la concentration spatiale des violences urbaines, ou encore, dans le cas particulier de Lima, la fragmentation administrative.

3 Les réformes néolibérales adoptées au tournant des années 1990 par l'ensemble des pays de la région ont par ailleurs eu comme résultat d'accentuer les inégalités sociales et ont participé de la radicalisation de la polarisation entre les élites et leurs marges, faisant des marges urbaines des symboles de l'insécurité. Considérées comme des foyers de délinquance et des zones de replis pour la criminalité, ces zones marginales ont été longtemps exclues de toute forme de planification urbaine et échappent encore en partie au contrôle territorial exercé par les États et collectivités locales. En relevant avant tout d'une construction sociale et spatiale ces marges, qui ne sont en outre pas nécessairement situées en périphérie, apparaissent comme l'expression d'une différenciation sociale exprimée par l'espace (Sierra, Tadié, 2008). Outre le creusement des inégalités, les réformes néolibérales ont eu pour conséquence la privatisation du service public, dont, entre autres, celle des activités policières. Certains auteurs, comme Wievorka, ont ainsi souligné les transformations caractérisant l'exercice de la violence légitime telle qu'elle est perçue dans sa conception wébérienne, alors que la police se voit de plus en plus assurée par des acteurs privés, entreprises ou collectivités d'individus (Wievorka, 2005).

4 Le vigilantisme reste un terme difficile à définir. De façon générale, une organisation vigilante apparaît comme un regroupement d'individus issus de la société civile mobilisés autour d'un objectif commun qui est généralement associé à la préservation et l'amélioration d'un système existant, ici celui instauré par la gestion de l'ordre public. Les membres de ces organisations prétendent agir en "dernier recours », et se présentent comme une alternative devant l'absence d'intervention de l'État (Hine, 1997). Johnston quant à lui définit le «vigilantisme » en tant que procédé par lequel la population organisée participe, voire s'empare, du maintien de l'ordre public (Johnston, 1996, p. 32). En Amérique Latine, le vigilantisme reste étroitement associé à des pratiques autoritaires et à l'usage de la violence (Huggins, 1991) À de nombreuses reprises, la prise en charge du maintien de l'ordre par la population a ainsi pu être détournée pour des raisons politiques et représenter des appuis non négligeables pour les forces de l'ordre traditionnelles, comme cela a pu être le cas au Pérou pour les Comités d'Autodéfense, inspirés des Rondes Paysannes, ou encore au Mexique, où l'incapacité de l'État à faire face à la violence du narcotrafic a provoqué la multiplication des lynchages et milices civiles (Fuentes Diaz, 2004).

5 Cet article cherche ainsi à interroger les modes de participation sociale aux activités développées par la police dans la métropole de Lima en s'attachant plus particulièrement à l'observation du rôle tenu par les Juntas Vecinales de Seguridad 
Ciudadana (JVSC), organisations vigilantes formées par des habitants d'un même quartier afin de seconder la police dans ses tâches de surveillance. Nous verrons dans un premier temps comment se forment et s'organisent les JVSC et dans quelle mesure on peut alors parler de coproduction du contrôle territorial entre la police nationale péruvienne, les municipalités et la population organisée. Nous passerons dans une deuxième partie à une analyse critique des JVSC, notamment sur la question de la légitimité de leur intervention sur le territoire. Ces analyses s'appuient sur un travail de terrain mené entre janvier 2012 et décembre 2013 dans la métropole de Lima-Callao dans le cadre d'un projet de recherche mené par l'équipe PACIVUR $^{1}$ sur les organisations sociales comme ressource de gestion de crise. Pour la réalisation de cette enquête, nous avons conduit des entretiens directifs et semi-directifs avec des coordinateurs de JVSC ainsi qu'avec les officiers de police collaborant avec eux. Nous avons pu aussi réaliser des observations participantes, particulièrement dans les districts liméniens de Villa El Salvador et La Victoria. Enfin, grâce à la collaboration du Général Yepez Dávalos, professeur à l'Institut des Hautes Études Policières à Lima ${ }^{2}$, nous avons pu réaliser un questionnaire parmi une trentaine de dirigeants de JVSC au niveau des districts.

\section{Les Juntas Vecinales de Seguridad Ciudadana : une police « citoyenne »?}

\section{La police péruvienne : une institution en transformation}

6 Au Pérou, la police apparaît comme une institution source de problème. Entre 1980 et 2000 , le pays traverse une période de crises économique et politique aigües, tandis que l'arrivée au pouvoir d'Alberto Fujimori en 1990 a contribué à favoriser les pratiques clientélistes au sein des institutions publiques. Le rétablissement de la démocratie en 2001 a signifié une véritable remise en question des institutions policières et des paradigmes de maintien de l'ordre. De nombreuses initiatives ont ainsi été adoptées par les gouvernements s'étant succédés depuis, ainsi que par les autorités locales. Le processus de décentralisation débuté au début des années 2000 a en outre contribué à favoriser un certain transfert des politiques de gestion de l'ordre public aux municipalités de district ${ }^{3}$, ce qui a permis l'intervention de nouveaux types d'acteurs. Les pouvoirs locaux peuvent ainsi s'appuyer sur une police municipale (serenazgo) qui, si elle ne dispose pas de mandat lui permettant de procéder à une arrestation, s'avère être une source d'appui important pour la police nationale (Costa, Romero, 2010, p. 113). Dans les quartiers les plus aisés, mais aussi de plus en plus dans ceux habités par les classes moyennes voire populaires, les entreprises de sécurité privées font par ailleurs recette en assurant la surveillance de quartiers résidentiels ou de sièges d'entreprises.

7 Ainsi au Pérou, un grand nombre de politiques publiques s'appuie sur la population organisée, alors que l'idée de "participation » apparaît comme la pierre angulaire des politiques sociales. Ce phénomène s'explique au travers de plusieurs facteurs. En premier lieu, il est nécessaire en effet de souligner l'importance acquise par les organisations sociales au sein même du fonctionnement urbain suite à la croissance exponentielle de Lima à partir des années 1940. L'aménagement du territoire urbain suite aux invasions de terre a été ainsi en grande partie réalisé en raison des 
mobilisations effectuées par les associations de migrants formés avant même l'installation (Bey, 1995). Dans un second temps, on peut supposer que les transformation des politiques de lutte contre la pauvreté dans les années 1990 dans les pays du Sud, et plus particulièrement en Amérique latine, notamment au travers des programmes de cash transfer mis en place par la Banque Mondiale ont contribué à faire émerger l'idée d'une participation des bénéficiaires, d'où le fameux concept d'empowerment (Lautier, 2001). Au Pérou, ces processus se produisent dans le cadre de l'instauration d'un régime autoritaire et clientéliste, avec pour conséquence une certaine dépolitisation de la participation sociale pour la restreindre à la recherche de solutions aux problèmes de survie quotidienne (Avila Moreno, Castellanos, 2003). Enfin, la démission d'Alberto Fujimori et le retour de la démocratie à la fin de l'année 2000 s'accompagnent de l'introduction du budget participatif au Pérou dans le cadre des lois sur la décentralisation et les collectivités territoriales qui terminent d'asseoir la légitimité d'une convocation de la population à la gestion des affaires publiques.

8 Si cela peut être considéré comme l'expression d'un libre exercice de la citoyenneté, cette forme de mobilisation des secteurs populaires et précaires de la société péruvienne n'est pas sans poser un certain nombre de problèmes, non seulement quant à sa légitimité, mais aussi par rapport aux relations de pouvoir qui se forment dans un tel contexte. Cette participation, ainsi que l'affirme Tanaka, est en effet avant tout le fruit d'une demande des pouvoirs publics et ne résulte pas nécessairement d'une initiative citoyenne spontanée comme certains discours, notamment ceux issus de la sphère politique, tendent à le faire croire (Tanaka, 2001, p. 6). Dans une métropole aussi fragmentée et étendue que Lima, l'engagement « citoyen » dans les politiques publiques est en outre susceptible d'accentuer les inégalités sociales, mais aussi de susciter de véritables conflits d'intérêts et des rivalités entre les différentes organisations participantes.

9 Cela est d'autant plus vrai que les politiques de gestion de l'ordre public participent elles aussi à l'appel lancé à la société civile de contribuer à la sécurité citoyenne. Depuis la fin des années 1990, les péruviens sont ainsi fortement encouragés par les pouvoirs publics à seconder la police dans ses tâches en formant des juntas vecinales de seguridad ciudadana ${ }^{4}$ (JVSC). La formation de telles organisations apparaît en ce sens en partie comme le corollaire urbain des Rondes Paysannes formées dans le cadre de la lutte contre le vol de bétail dans le Nord-Ouest du Pérou (Perez Mundaca, 1996) ou encore des Comités d'Autodéfense censés seconder l'armée dans la lutte contrerévolutionnaire menée contre le Sentier Lumineux. Ces milices paysannes, qui ne font l'objet d'une véritable reconnaissance juridique qu'en $1991^{5}$, ont joué un rôle prédominant dans la capitulation des groupes armés mobilisés contre l'État depuis les années 1980 (Degregori, 1996). Or s'il est possible d'établir des liens entre ces deux organisations dans le cadre d'un encouragement implicite des pouvoirs publics à lutter contre la subversion politique, alors devenue synonyme d'insécurité, les JVSC et les Comités d'Autodéfense restent cependant deux types d'organisation différentes l'une de l'autre en raison de nombreux facteurs. Le principal est leur partenaire public : alors que les Comités d'Autodéfense dépendent essentiellement du Ministère de la Défense et continuent de justifier leur existence au travers de leur contribution à la lutte " antiterroriste " menée dans les régions centrales du pays, les JVSC, quant à elles, entretiennent des relations avec le Ministère de l'Intérieur (plus précisément avec la Police Nationale) et sont présentes sur l'ensemble du territoire national. Ces deux types d'organisations apparaissent donc à la fois comme étant complémentaires et rivales 
alors qu'elles font intervenir deux acteurs clefs de la sécurité publique au Pérou: l'armée et la police.

10 En ce sens, la mobilisation de la population permet de pallier le manque de moyens dans les territoires où les municipalités n'ont pas les ressources pour financer une police municipale ou une sécurité privée. Elle représente par ailleurs une opportunité de rapprochement des institutions policières avec la société civile. Si la participation sociale aux activités de police peut, sous certains angles, apparaître comme une réponse alternative aux problèmes de sécurité publique, elle n'est pas sans soulever à son tour un certain nombre d'interrogations quant à la légitimité réelle du contrôle territorial exercé par de telles organisations et à la reproduction des inégalités dans la réalisation du service public.

\section{Formation et institutionnalisation des Juntas et coproduction du contrôle territorial à Lima.}

11 Les premières JVSC voient le jour à Lima à la fin des années 1990 et peuvent être analysées comme l'expression d'un effort de rapprochement de la PNP (Police Nationale du Pérou) avec la société civile. Le Pérou se trouve en effet à ce moment-là en pleine transition politique. Si le spectre de la violence armée a commencé à s'effacer, le régime autoritaire mis en place par Fujimori depuis le début des années 1990 s'appuie sur des pratiques clientélistes portant atteinte à l'intégrité des institutions publiques. Les liens étroits unissant la police et l'armée, le recours à l'intervention de groupes paramilitaires et l'utilisation des forces de l'ordre dans la répression de la protestation sociale représentent autant de facteurs qui ont contribué à éroder la confiance des Péruviens en leur police. Par ailleurs, si le retour de la démocratie à la fin de l'année 2000 a bien signifié (en théorie) la réhabilitation d'un État de droit, la publication en 2003 du rapport final de la Commission Vérité et Réconciliation qui met en cause les forces de l'ordre (l'armée, mais aussi la police) dans les violations des droits humains ayant eu lieu pendant le conflit armé des années 1990, n’a guère contribué à une réhabilitation de l'institution ${ }^{6}$.

La police péruvienne apparaît cependant comme une institution en pleine mutation depuis la réunification des trois institutions la composant en $1988^{7}$ et le développement du concept de "sécurité citoyenne" dans l'ensemble des pays d'Amérique latine (Carillo Florez, 2007). La police par ailleurs, de par la nature même de ses fonctions, représente un instrument de pouvoir indéniable en étant au contact direct des masses populaires. Cela permet en outre de contourner les mouvements sociaux susceptibles de remettre en question le régime instauré par Fujimori depuis le début des années 1990. En effet, si le contexte de crise politique aigüe induit par le conflit armé a contribué à décourager toute remise en question du pouvoir, le retour progressif de la paix à partir du milieu des années 1990 s'accompagne d'une visibilité croissante des associations de défense des droits humains à l'échelle internationale qui apparaissent comme autant d'éventuelles menaces au maintien du régime (Yongers, 2003). L'une des stratégies de Fujimori afin de contrer l'opposition est d'établir une relation directe avec les couches populaires, notamment dans les marges urbaines. C'est dans ce contexte qu'apparaissent les premières initiatives et infrastructures qui permettent le rapprochement entre police et citoyenneté. Alors que ce phénomène tend à s'observer sur l'ensemble des pays de la région et qu'il est plutôt considéré comme synonyme de 
démocratie, au Pérou il se présente au contraire comme une stratégie politique permettant le maintien au pouvoir d'un gouvernement autoritaire (Basombrio, Rospigliosi, 2006). Malgré cela, on assiste à la fin des années 1990 à un véritable effort d'articulation de la PNP avec la population, notamment par la création d'une Direction de Participation Citoyenne ${ }^{8}$ en 1997 à l'initiative du général de police Enrique Yepez, à l'origine d'une véritable institutionnalisation des liens de collaboration entre la police péruvienne et la société civile. Le général Yepez est en effet l'instigateur des JVSC, qui, contrairement à ce que l'on pourrait penser, ne sont pas nées de façon spontanée, mais répondent à une demande des institutions publiques poussées à rechercher la participation sociale en raison des nouvelles doctrines économiques dictées par les organisations internationales au tournant des années 1990. Les JVSC sont formées en premier lieu dans les quartiers périphériques de Lima, territoires étendus et informels que la police a du mal à contrôler par manque d'information et de moyen, alors que les quartiers centraux et formels font l'objet d'une attention plus grande et plus ancienne Elles sont donc composées par des habitants des différents quartiers qui rencontrent des problèmes de sécurité publique, en raison de l'absence d'État. Nés d'invasions de terrains, la majorité de ces quartiers ne dispose pas des infrastructures essentielles (raccordement électrique, eau courante, routes asphaltées, collecte des eaux usées) au fonctionnement urbain. Ils apparaissent ainsi comme des zones de «non droit » au sens strict du terme, dans la mesure où, en effet, il s'agit d'espaces informels situés en marge des administrations urbaines officielles. Les associations formées par des groupes de voisins, dont les familles sont généralement issues des mêmes villages, se présentent ainsi comme les moteurs de l'incorporation de ces nouveaux quartiers à la métropole, jouant le rôle d'intermédiaires entre les pouvoirs publics et les habitants. Il existe ainsi dans ces quartiers une certaine tradition de l'organisation collective qui facilite l'expérimentation de nouvelles formes d'articulation entre habitants organisés et pouvoirs publics.

C'est pourquoi les institutions policières ont cherché à former les JVSC avant tout dans ces quartiers, d'une part parce qu'il existe un intérêt stratégique pour la police à étendre son influence sur un territoire qu'elle contrôle peu ${ }^{9}$, d'autre part parce qu'il existe depuis longtemps dans ces quartiers une gestion collective du territoire, prérequis nécessaire à de telles organisations. Cette initiative s'étend cependant peu à peu à l'ensemble de la métropole (composée de 43 districts pour Lima et de 6 pour le Callao). En 2012, entre 4500 et 5000 JVSC étaient recensées par les Bureaux de participation citoyenne des régions policières de Lima et $\mathrm{Callao}^{10}$. Dans la mesure où ils émanent de la police, ces chiffres sont à manier avec précaution, puisque volontiers gonflés par les officiers responsables du recensement des JVSC. Malgré cela, il est possible de dire qu'entre 40000 et 50000 personnes participent à ces organisations de voisins vigilants sur l'ensemble du territoire métropolitain. Leur articulation avec la police leur permet en outre d'être présentes sur l'ensemble du territoire et une organisation hiérarchique d'être représentées à tous les niveaux de gouvernement ${ }^{11}$.

\section{Le fonctionnement des juntas et leurs limites matérielles et symboliques}

14 Les initiatives de rapprochement des institutions policières de la population se multiplient à la fin des années 1990 avec notamment la création en 1997 des «bureaux de participation citoyenne » (OPC - Oficina de Participacion Ciudadana) dont doivent être 
pourvus chaque commissariat du pays, ainsi que les directions policières territoriales. Placées sous l'autorité du commissaire, ces OPC sont tenues par des officiers ${ }^{12}$ de police chargés spécifiquement d'organiser les activités proposées par la police à la communauté. Il s'agit de campagnes d'information de santé publique (cela va de la coupe de cheveux gratuite aux vaccins pour les enfants), de jeux pour les plus jeunes, de manifestations sportives etc. Parmi les activités développées par les OPC figure en outre la participation citoyenne à la sécurité publique, sous la forme des JVSC (mais pas exclusivement) qui vont être articulées aux différentes échelles du territoire. Au niveau local cependant, leur institutionnalisation relève du commissariat de quartier. En effet, si les JVSC sont considérées comme des organisations autonomes, les chargés d'OPC (dits aussi "promoteurs») ont pour mission de les inscrire dans un registre qu'ils conservent au commissariat, de choisir leurs représentants en accord avec les membres de l'organisation, d'animer les réunions entre les JVSC et les autres membres du commissariat dont le commissaire ainsi que de proposer des patrouilles mixtes entre membres des JVSC et policiers. C'est aussi à eux qu'il revient de former les JVSC aux tâches de surveillance, en échange d'une contribution de ces dernières aux activités sociales menées par la police dans les quartiers.

Les JVSC de leur côté se présentent comme des organisations volatiles. En théorie une personne qui souhaite participer doit accepter d'être inscrite dans le registre tenu par le commissariat, mais en pratique les membres vont et viennent à leur guise et il est difficile de réellement se faire une idée du nombre de personnes mobilisées à long terme. Néanmoins, la structuration de l'organisation autour des institutions policières leur offre une certaine stabilité. En effet, les JVSC suivent le découpé territorial de la police péruvienne, légèrement différent de celui opéré par les administrations civiles, ce qui leur donne une certaine autonomie vis-à-vis des municipalités. Chaque district liménien procède à son propre découpage territorial en identifiant les différents secteurs le constituant. Ces secteurs sont à leur tour divisés en manzanas (blocs résidentiels) ou zones. Les commissariats implantés dans le district étendent ainsi leur autorité sur un nombre déterminé de secteurs, sachant qu'il y a en général plusieurs commissariats par district. Mais dans certains cas, un commissariat peut exercer son contrôle territorial sur un ensemble de secteurs relevant de plusieurs districts. C'est le cas par exemple pour le Commissariat de Yerbateros situé dans le district d'Ate Vitarte dans l'Est de la capitale, qui étend ses compétences sur quelques secteurs des districts voisins de La Victoria, d'El Augustino et du Cercado. De façon générale, les JVSC s'intègrent au sein d'une administration dont les compétences d'intervention sur le territoire dépassent les limites de districts, ce qui leur permet de se différencier des autres types d'organisations sociales mobilisées dans les quartiers qui répondent en général avant tout à la municipalité.

Une junta est ainsi généralement formée par les habitants d'une même manzana, qui ont à leur tête un coordinateur. Ce dernier, censé assumer cette fonction sur une durée de deux ans, fait alors l'objet d'une inscription au registre tenu par l'OPC du commissariat de quartier et se voit à son tour chargé de nommer un responsable de secteur, c'est-àdire d'une zone regroupant plusieurs manzanas. Ce sont les coordinateurs de secteurs qui entre autres fonctions, se rendent aux réunions régulières organisées au commissariat d'où dépend la JVSC, au cours desquelles est déterminée la marche à suivre pour l'ensemble des JVSC recensées au commissariat. C'est aussi au cours de l'une de ces réunions que va être nommé le coordinateur de commissariat, chargé de 
représenter l'intermédiaire entre ce dernier et l'ensemble des JVSC mobilisées sur l'ensemble du district. Nommé par le commissaire, ce coordinateur doit néanmoins pouvoir compter sur la collaboration des coordinateurs de secteurs afin de pouvoir agir et sa nomination par le commissaire fait le plus souvent l'objet d'une consultation entre les différents acteurs impliqués, les policiers comme les membres de JVSC. Enfin, entre l'ensemble des commissariats d'un seul et même district est nommé un coordinateur dit « de district » qui sera de son côté amené à participer aux réunions se tenant au niveau de la région PNP, c'est-à-dire Lima ou Callao, qui ont lieu en moyenne deux fois par mois. Ainsi, à chaque niveau de déploiement des JVSC correspond un OPC et un officier de police chargé d'établir la liaison, ce qui permet une diffusion des informations sur l'ensemble de la métropole.

Toute personne assumant une responsabilité de coordination au sein d'une JVSC fait l'objet d'une inscription au registre tenu par l'OPC du commissariat de quartier et se voit attribuer une carte d'identification mentionnant l'identité du porteur et son rôle dans la junta. Chaque coordinateur doit par ailleurs être en mesure de présenter un casier judiciaire vierge. Dans certains cas, la formation de nouvelles JVSC, qui suppose donc la désignation de nouveaux coordinateurs fait l'objet de cérémonies officielles au cours desquelles les nouveaux adhérents prêtent serment. Cette pratique néanmoins n'est pas systématique lors de la constitution d'une junta et a avant tout pour objet la promotion de ce type d'organisation dans les quartiers encore peu mobilisés. À l'heure actuelle, sur la région policière Lima (c'est-à-dire l'ensemble des districts) plus de 46000 personnes participeraient à l'une des 4583 JVSC recensées par l'OPC - Lima ${ }^{13}$.

Les JVSC mènent différents types d'activités. La plus importante consiste à rapporter des informations à la police sur les zones à risques de leur quartier, où ont eu lieu un vol, une agression, une vente ou consommation de drogue, une fête illégale, l'ouverture d'un commerce en dehors des horaires autorisés etc. Ces organisations se révèlent ainsi de véritables outils de cartographie de la délinquance et de l'insécurité à Lima-Callao dans la mesure où elles permettent de les localiser. Bien souvent d'ailleurs, la limite entre vigilance et espionnage est extrêmement fine et les membres des juntas ne sont pas nécessairement vus d'un bon œil par tous les riverains. Dans certains districts comme Villa El Salvador, chaque secteur de JVSC possède son propre local (cela reste cependant exceptionnel), ce qui en fait de véritables appuis pour la police. Ces locaux sont en général mis à disposition par la municipalité et leur entretien est à l'entière charge des riverains et membres des JVSC qui ne touchent pas ailleurs aucune forme de compensation pour le temps passé à déployer leurs activités de surveillance et de cohésion sociale. Outre les patrouilles régulières, d'autres types d'activités sont réalisés conjointement par les JVSC et la police. Alors qu'elles ne comptent sur aucune forme de subventions publiques, les JVSC fonctionnent avec un budget extrêmement limité. Dans la plupart des districts, les membres de JVSC sont équipés de sifflets et de gilets permettant leur identification, matériel qu'ils utilisent lorsqu'ils réalisent leurs patrouilles ou lorsqu'ils participent à un événement de leur quartier. Ces patrouilles ont lieu de façon plus ou moins régulières selon la junta mais il est raisonnable de dire qu'elles sont au moins hebdomadaires, voire quotidiennes dans certains districts. Lorsque les relations entre les JVSC et la municipalité sont bonnes, les gilets et sifflets sont parfois financés par cette dernière. Le plus souvent, ce sont les membres des juntas eux-mêmes qui mettent la main à la poche afin de s'équiper. La police de son côté en effet ne procure aucun support matériel au JVSC. Son rôle consiste à apporter son soutien tant dans les tâches de surveillance que de formation. C'est avant tout grâce à 
la solidarité des voisins et la bonne volonté de leurs propres membres que les JVSC réussissent à maintenir les conditions leur permettant d'exercer leurs activités. Le plus souvent, un petit budget est réuni grâce à l'organisation de polladas, sortes de barbecues de quartier, une pratique extrêmement courante au Pérou. Les bénéfices sont alors utilisés pour une cause généralement connue à l'avance (payer une opération chirurgicale onéreuse, financer un projet associatif, payer un enterrement etc.). Dans certains districts par ailleurs, les JVSC réussissent à établir des accords avec certaines entreprises privées qui leur cèdent du matériel ou des outils de communication leur permettant de développer leurs activités.

Les JVSC représentent donc avant tout d'un intermédiaire entre la population et la police et légitime cette dernière en l'assistant par ailleurs dans les activités qu'elle réalise au sein de la communauté, en jouant notamment le rôle d'organisateurs. En ce sens elles représentent un soutien important pour la police en l'accompagnant dans ses tâches de développement social. Il est ainsi possible de parler d'un accompagnement mutuel des juntas et de la police : au-delà des patrouilles mixtes régulières, c'est aussi bien souvent ensemble qu'elles participent ou organisent les événements marquant la vie collective de leurs quartiers. En ce sens les JVSC représentent une véritable ressource sociale pour la police qui peut s'appuyer sur elles afin de gagner la confiance de la population et maintenir une certaine autorité.

Les juntas cependant ne représentent cependant pas seulement des ressources symboliques. Ainsi, pour reprendre l'exemple de Villa El Salvador, le coordinateur de district est toujours très fier de faire visiter les nouveaux dortoirs récemment construits pour les policiers au sein du commissariat central ${ }^{14}$. Les travaux avaient été entièrement menés et financés par la société civile, par le biais de l'association "des amis de la police » et le soutien d'entreprises privées (dons de matelas, de matériel de construction etc.). Si l'association formée par les « amis de la police » n'est pas la même que celle des JVSC, on retrouve bien souvent les mêmes membres. Cet exemple montre plusieurs choses. D'une part il met en évidence le manque de moyens donnés par un État à sa police nationale. D'autre part, il illustre les relations étroites nouées par la police avec certains habitants et souligne les éventuels liens d'allégeance qui peuvent en résulter. Si elles servent les intérêts de la police nationale en lui permettant de s'introduire dans certains quartiers difficiles d'accès, les JVSC se présentent en effet avant tout comme des organisations disposant d'une certaine autorité non seulement sur la police mais aussi sur leurs concitoyens. 


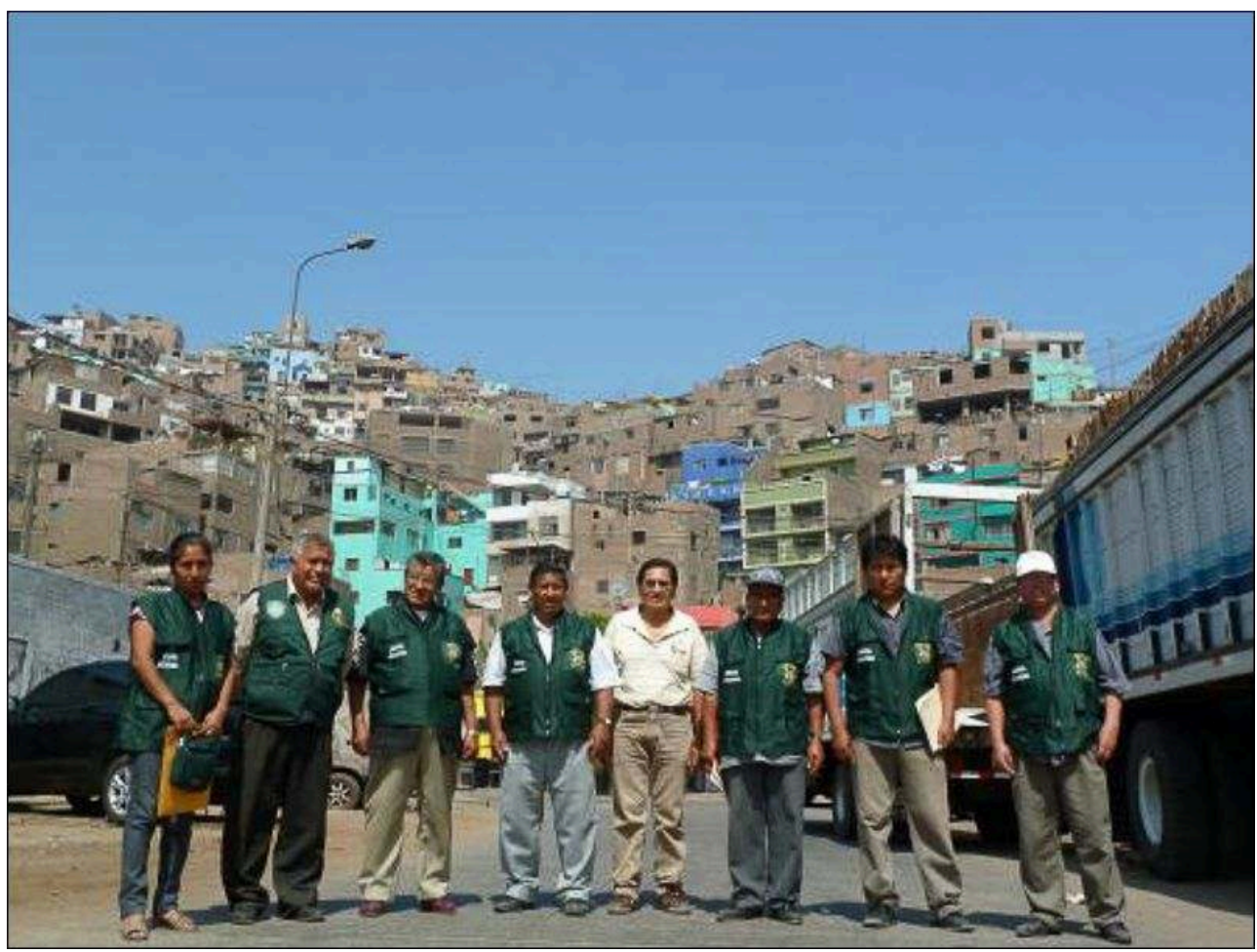

Source : Ideele

\section{La Junta et le commissariat. Une ressource du fonctionnement urbain?}

\section{Les Juntas à l'origine d'un soutien populaire à la police de quartier}

21 Les coordinateurs de JVSC répètent régulièrement qu'ils «sont capables de virer un commissaire corrompu ». Quintiliano Ponce, représentant des JVSC sur l'ensemble du territoire national et habitant de Villa El Salvador, raconte ainsi avoir, du temps où il était dirigeant de son district, réussi à faire muter un commissaire qui refusait de collaborer avec les juntas ${ }^{15}$. Suzano, lui-même coordinateur de JVSC pour le quartier informel de cerro el Pino, situé sur un pan de montagne au milieu du district de La Victoria, affirme quant à lui avoir réussi à convaincre la haute hiérarchie policière de "leur laisser leur commissaire un an de plus ${ }^{16}$ ». Nommés en début d'année civile, les commissaires sont en effet généralement mutés après un an d'exercice sur d'autres affectations, en vue notamment d'éviter la corruption. Si ce n'est pas le cas des officiers présents dans les OPC, généralement en poste plus longtemps, c'est parce que ces mutations compliquent les relations entre la police et les JVSC qui préfèrent, lorsque cela se passe «bien», garder «leur» commissaire. Cette notion d'appropriation du commissaire semble par ailleurs assez répandue parmi les membres actifs de JVSC, du moins chez ceux qui sont amenés à exercer un certain leadership et à interagir régulièrement avec la police. On la retrouve notamment dans les paroles de Julia Cahua, élue coordinatrice de l'ensemble des JVSC sur les 43 districts de Lima depuis septembre 2012, qui, lorsqu'on lui propose d'organiser des réunions ou ateliers de travail entre notre équipe et les coordinateurs de districts de JVSC, invoque 
régulièrement la nécessité d'en parler avec «son » colonel, c'est-à-dire le responsable d'OPC pour la $7^{\mathrm{e}}$ région policière (Lima) ${ }^{17}$.

Chaque district de Lima-Callao possède un représentant de JVSC, même dans le cas où, comme on peut l'observer dans les zones les plus cossues de la métropole, il ne dispose guère de « base » au niveau local. Ce sont en effet avant tout les résidents des quartiers populaires qui tendent en majorité à participer au maintien de l'ordre public. La participation aux JVSC est bénévole et souvent ingrate dans la mesure où elle nécessite de veiller tard le soir et inspire de la méfiance du voisinage. Suzano raconte ainsi avoir été inquiété à de nombreuses reprises, tant et si bien que sa femme a menacé de le quitter ${ }^{18}$. Quintiliano de son côté travaille à Barranco (un district relativement proche de Villa El Salvador) et consacre tout son temps libre à la formation de nouvelles JVSC sur tout le territoire national, ainsi qu'à l'appui de la police dans les campagnes de prévention de la délinquance. Tous deux ne sont pas originaires de Lima mais viennent des zones andines; Suzano Enciso notamment vient de Quinua un village situé près d'Ayacucho, l'une des régions les plus touchées par le conflit armé. Migrants de première génération, Quintiliano et Suzano ont fondé leur foyer à Lima où ils se sont installés, par « invasion ", dans les années 1970. Tous deux ont un de leurs enfants parti étudier à l'étranger.

Moins âgée que ses homologues de Villa El Salvador et La Victoria (qui ont près de soixante-dix ans) Julia Cahua, une quarantaine d'années, n'en partage pas moins certains traits avec eux. Elle aussi originaire de province (côte nord du Pérou), elle affirme de son côté se dédier "à plein temps » à la junta, tandis que Quintiliano Ponce et Suzano Enciso travaillent tous deux (l'un tient un commerce, le second est mécanicien dans un garage). D'après elle, sa fonction de coordinatrice des quarantetrois districts liméniens ne lui permet pas en effet d'exercer une « autre profession ${ }^{19}$ ». Elle ne précise cependant pas d'où elle tire ses ressources, d'un membre de sa famille ou par le biais d'une rémunération "alternative » (biens matériels, alimentation, bons de transport) obtenue en raison de son implication au sein des JVSC. Et si, selon le recensement établi par la police nationale, les femmes représentent près de la moitié de la totalité des membres des juntas, elles sont sensiblement moins représentées dès qu'il s'agit de monter dans la "hiérarchie ». Le fait qu'une femme soit coordinatrice pour l'ensemble de Lima Métropolitaine est donc, en soi, l'expression d'un certain volontarisme.

Il existe ainsi de la mobilité sociale dans la trajectoire de ces trois acteurs qui sont assez représentatifs du leadership des JVSC. Malgré leur articulation étroite avec la police, ces organisations réussissent à conserver une autonomie, voir même à exercer une forme de contrôle sur les institutions policières, ce qui leur permet de bénéficier d'une certaine légitimité auprès du public. En ce sens, elles jouent un véritable rôle représentatif et permettent une certaine inclusion de quartiers marginalisés tout en rendant visibles aux yeux des autorités des secteurs sociaux précaires. Les membres des juntas peuvent cependant irriter parfois leurs voisins en se montrant trop pointilleux dans leurs tâches. Il s'agit en effet pour beaucoup d'entre eux de retraité(e)s, la plupart ayant grandi en province et ayant migré à Lima à la fin des années 1970, dont les mœurs peuvent être en décalage avec ceux des jeunes de leur quartier, ce qui provoque éventuellement des frictions. Il n'en reste pas moins que leur capacité à s'imposer face à la police est généralement plutôt appréciée. En ce sens, les JVSC représentent de véritables espaces d'affirmation sociale. Au niveau individuel, et à l'instar d'autres 
organisations populaires à Lima-Callao, elles représentent des plateformes de mobilité sociale pour des individus issus des secteurs les plus populaires. Des individus comme Quitiliano Ponce, Suzano Enciso ou Julia Cahua ont su mettre à profit en effet le réseau créé par leurs activités au sein des JVSC pour poursuivre leur carrière "de dirigeants » populaires en assumant d'autres charges, soit au sein de la direction nationale des JVSC, comme c'est le cas de Quintiliano Ponce, soit dans d'autres types d'organisations. À l'échelle collective, les juntas participent activement au contrôle territorial exercé par l'État. Elles permettent de rééquilibrer une organisation des rapports sociaux qui, concrètement et symboliquement, est orienté par des dynamiques de race, de classe et de sexe. Ces facteurs tendent en effet à structurer la société péruvienne et représentent les premiers facteurs de vulnérabilité de la population.

Les JVSC apparaissent donc comme un instrument d'affirmation des classes populaires ; ce qui confirme par conséquent l'idée qu'elles permettent une forme de démocratisation des politiques de gestion de la sécurité publique. Par ailleurs, en se présentant dans des quartiers comme les principaux intermédiaires entre la population et les pouvoirs publics, et dans certains cas avec les ONG, elles incarnent de véritables outils de gestion du territoire, voire de développement. A titre d'exemple on peut citer l'Institut de Défense Légale, une importante ONG péruvienne qui possède de longue date un programme sur la sécurité citoyenne et qui travaille très régulièrement avec les JVSC dont elle tente de valoriser le rôle. Selon le responsable du programme, Cesar Bazán, les juntas « sont la clef » pour l'amélioration de la sécurité (Bazan, 2013).

Les juntas ne sont pas cependant sans rencontrer certaines limites et poser quelques problèmes. Si elles disposent bien d'une réelle légitimité, non seulement auprès de la société civile, mais aussi des autorités publiques, il n'en reste pas moins vrai qu'elles sont à même d'exercer certains abus. Par ailleurs, le succès, rappelons-le différent selon le district, des JVSC cache une certaine incapacité de l'État à réduire les inégalités sociales.

\section{Vers la formation de nouveaux espaces de vigilance ? Pouvoirs concurrentiels et disputes du contrôle territorial}

Concernant le fonctionnement des JVSC, il existe un règlement édicté par la police. $\mathrm{Si}$, comme mentionné plus haut, il s'agit bien d'organisations autonomes, leur raison d'être les lie étroitement aux institutions policières au travers de relations qui peuvent contourner l'État de droit. Il est ainsi représentatif que Suzano Enciso et Quintiliano Ponce, en différents moments chacun, aient raconté avec une certaine fierté les « rafles » de fumones ${ }^{20}$ et « d'homosexuels » (jeunes hommes prostitués) auxquelles ils auraient participé en 2002. Suzano évoque ainsi avoir monté une opération avec les autres coordinateurs des juntas de La Victoria afin de "nettoyer» le quartier des délinquants. Une nuit, ils auraient loué une dizaine de taxis et auraient séquestrés quatre-vingt vendeurs de drogues et les petits chefs de bandes, qu'ils auraient emmenés loin du quartier, dans le nord de Lima où il existait encore à l'époque des terres agricoles. Là, ils les auraient battus, dévêtus et laissés sur place, "pour qu'ils $y$ restent et ils ne sont pas revenus, croyez-moi, après deux mois seuls deux d'entre eux sont revenus ${ }^{21}$ ». La police n'aurait pas participé à cette opération totalement illégale. Les JVSC n'ont officiellement pas le droit de mener une opération « de force » sans l'appui de la police nationale (ce genre d'intervention violente bafoue d'ailleurs les droits les 
plus élémentaires). On peut supposer cependant que la police ait été tenue informée de l'intervention, même à posteriori, et qu'elle ait décidé de fermer les yeux sur son caractère illégal. Quintiliano Ponce, selon ses dires, a lui aussi participé à une rafle, à peu près à la même époque, à Villa El Salvador ${ }^{22}$.

Ces exemples sont emblématiques du caractère flou de la légitimité du contrôle territorial exercé par les JVSC. Si de telles opérations ont très certainement recueilli l'approbation des riverains qui se sont vus, du jour au lendemain, « débarrassés » d'un nombre significatif de délinquants, au niveau légal, elles sont extrêmement problématiques, d'autant plus lorsqu'elles comptent sur l'indulgence de la police qui tend parfois à fermer les yeux face à de tels actes. En février 2012, lors d'entretiens avec les JVSC, plusieurs membres racontent fièrement comment ils ont "attrapé " un délinquant et l'ont laissé "pendu » à un réverbère, au vu et su de tous, durant toute une nuit avant de le libérer. Bien que cet acte soit illégal, la police n'a pas cherché à sanctionner les membres des JVSC à l'initiative de cette humiliation publique. Les JVSC exercent donc un véritable contrôle physique des personnes présentes sur leur territoire, ce qui n'est pourtant pas prévu au sein de leur règlement de fonctionnement, et elles peuvent éventuellement contourner les lois sans être réprimandées par la police. Il y a donc bien un système d'échange de services entre cette dernière et les juntas, ainsi que le partage d'un territoire. Or même si les juntas recueillent dans la plupart des cas l'approbation des riverains, ou en tout cas de ceux qui dominent l'espace public de leur quartier, ces actes restent en marge des lois. En ce sens les JVSC sont assez représentatives de ce que Goldstein identifie comme la "justice flexible ", lorsqu'il se réfère dans le cas bolivien aux stratégies « d'autodéfense » adoptées par les organisation vigilantes afin de faire face aux failles induites par l'État (Golstein, 2005, p. 396).

Il existe par ailleurs une certaine rivalité entre les JVSC et les autres organisations sociales mobilisées dans les quartiers populaires de Lima. En étant associées à la police nationale, les juntas sont intégrées à une chaîne de commandement qui s'articule sur les différentes échelles du territoire. Cela représente une certaine spécificité dans le cadre d'une métropole caractérisée par son extrême fragmentation administrative où les pouvoirs municipaux bénéficient d'une grande autonomie et agissent en territoires indépendants. Ainsi, depuis 2003 les municipalités de Lima ont créé leurs propres associations de voisinage qui se posent comme des concurrentes directes des JVSC patronnées par la police nationale. Élues par les riverains sur des listes composées par secteur et validées par la municipalité, ces associations ont aussi pour but de participer à l'amélioration de la sécurité de leur quartier. Elles se consacrent cependant plus concrètement à des tâches relevant avant tout de la gestion du territoire, comme l'organisation de journées de nettoyage des espaces publics, la définition des politiques d'urbanisme, la création des budgets participatifs, etc. Des tensions palpables existent entre les deux types d'organisation. Les JVSC se voient en effet bien souvent négligées par les maires de districts qui leur préfèrent leurs homologues municipales avec lesquelles ils entretiennent de meilleures relations.

Cette mésentente entre les JVSC et les pouvoirs municipaux est en réalité assez fréquente. Ainsi, tandis que chaque municipalité de Lima-Callao est chargée d'établir un plan de " sécurité citoyenne » en convoquant plusieurs acteurs emblématiques de la communauté (police, fonctionnaires municipaux, représentants de l'Église Catholique, dirigeants d'organisation sociale), il est intéressant de voir que les responsables de 
juntas sont rarement sollicités et qu'on leur préfère dans bien des cas leurs « frères ennemis ». Martha, coordinatrice d'une petite trentaine de JVSC pour l'ensemble du district de Breña, dans le centre de Lima, se plaint ainsi d'avoir été écartée du CODISEC (Comité Distrital de Seguridad Ciudadana), le conseil municipal chargé d'établir les stratégies de sécurité publique dont les membres sont nommés par le maire ${ }^{23}$. Elle affirme que ce dernier aurait mis à sa place une dirigeante sociale appartenant aux associations de voisinage concurrentes et adhérente du parti politique au pouvoir ${ }^{24}$.

Les rivalités caractérisant les institutions policières et municipales ne sont pas les seuls facteurs de vulnérabilité des JVSC. Ces dernières apparaissent aussi comme les expressions des très fortes inégalités caractérisant l'ensemble de l'organisation urbaine liménienne. De fait, si les commissariats semblent répartis de façon relativement homogène sur le territoire, en réalité ce sont avant tout les quartiers riches qui ont à leur disposition le plus de ressources policières. «Il y a une inégalité, mais pas tant de la faute du commandant, sinon que dans les zones de commissariats $A$ et $B$ [élites économiques] vivent des fonctionnaires de haut niveau qui ont besoin d'avoir des policiers près d'eux. Il y a des ambassades, bref en faisant la somme de tout ça, ça fait plein de policiers $»^{25}$. Ces paroles du général Yepez illustrent le problème politique caractérisant l'attribution des ressources en personnel, infrastructures et matériels utilisés dans le cadre du maintien de l'ordre. Les districts les plus riches sont ainsi ceux concentrant le plus de policiers et de matériels (véhicules, ordinateurs, armes) alors que ce sont paradoxalement eux qui disposent de plus de moyens pour engager une police municipale, dans la mesure où ils disposent de revenus suffisants grâce au prélèvement des impôts locaux. Les JVSC apparaissent donc là où la police est, sinon absente, du moins démunie. Elles traduisent les inégalités qui structurent la société péruvienne et accentuent la fragmentation territoriale et sociale de Lima-Callao. Dans un tel contexte les JVSC, en secondant, voir en remplaçant la police sur certaines portions du territoire urbain, disposent d'une certaine marge de manœuvre, qui tend à augmenter d'autant plus que les ressources dont dispose le commissariat local sont faibles.

En 2004, Lima comptait un policier pour 1200 habitants (Yepez Davalos, 2004, p. 149-151). Dans un tel contexte, la police est tenue de tolérer les pratiques de justice populaire de certaines JVSC. Ainsi que le souligne Lossio, les coutumes de lynchage qui s'observent en milieu urbain résultent de plusieurs facteurs dont principalement les carences matérielles et la faiblesse, ineffectivité ou/méconnaissance des lois (Lossio Chavez, 2008, p. 134). Si les juntas peuvent se présenter comme des alternatives au manque de ressources allouées à la police nationale, elles ne représentent en rien la solution au problème posé par les causes profondes de ces inégalités.

\section{Conclusion}

Le concept de "sécurité citoyenne » tend à présenter la sécurité comme un droit. Il est finalement plus intéressant, à l'instar de Carrillo-Flórez, de considérer cette dernière comme un bien (Carillo Florez, 2007, p. 182). Les réformes néolibérales adoptées dans les années 1990 ont eu pour conséquence un véritable retrait des services publics qui se sont vus remplacés, selon les cas, par le secteur privé ou par l'action collective. En d'autres termes, la participation sociale aux activités de police en tant que telle apparaît avant tout comme l'expression de fortes inégalités sociales. Comme le souligne Plöger, l'insécurité reflète les inégalités sociales, tout comme le manque d'accès aux 
services publics de base et l'équipement en infrastructures (Plöger, 2006). La participation sociale aux activités policières à Lima relève cependant avant tout des profondes inégalités observables dans la société péruvienne, bien plus que d'une précarité de moyens. Alors que l'idée de "participation » est encensée par l'ensemble des discours politiques sur la démocratie et la citoyenneté, elle cache en réalité l'incapacité, ou l'indifférence, des classes dirigeantes à rééquilibrer les modes d'accès à l'ordre public.

Ainsi, tandis que les groupes sociaux les plus aisés sont en mesures de faire appel au secteur privé, tout en étant de facto privilégiés par les pouvoirs publics dans la répartition des ressources policières, les plus pauvres ont pour alternative l'action collective qui, si elle peut se révéler un véritable moteur d'émancipation et d'affirmation sociale, est aussi susceptible de reproduire des rapports de domination au sein même des groupes sociaux marginaux.

D'un autre côté, rappelons que les organisations populaires ne sont pas toutes, loin s'en faut, nées de mouvement spontanées, mais ont fait l'objet d'un demande dans certains cas. Cette logique d'offre et de demande de l'action collective nous amène à nous interroger sur ses différents modes de contrôle et ce à différentes échelles, du régional vers le municipal, du municipal vers le quartier, mais aussi entre différents acteurs, de l'homme politique influent au policier, du policier au dirigeant populaire, du dirigeant populaire à ses voisins etc. L'analyse de la participation sociale aux activités de police à Lima nous permet ainsi, non seulement de mettre en évidence le rôle joué par l'espace dans la répartition des sphères d'influence entre les acteurs impliqués dans la gestion de l'ordre public, mais aussi de penser la reproduction des inégalités au travers du prisme de la marginalité urbaine.

Enfin reste à souligner que l'investissement de la population dans la gestion de la sécurité publique n'est pas un phénomène nouveau. La participation citoyenne aux activités de police doit être lue aussi bien comme un résultat des réformes néolibérales ayant empiété sur le monopole de l'exercice de la violence légitime par l'État et un phénomène général de privatisation de la gestion de l'ordre public, que comme l'imposition d'un modèle transnational de contrôle social (Pratten qud Sen, 2008).

Avila Moreno J., Castellanos T., 2003. Nuevos movimientos sociales y segregacion urbana en Lima Metropolitana. Ciudadania y Democracia, $\mathrm{n}^{\circ} \mathrm{1}$, Lima, Alternativa. Centro de Investigacion Social y Educacion Popular.

Basombrio C., 2007. Delito e inseguridad ciudadana. Lima y otras ciudadades del Peru comparadas con América Latina. Lima, Instituto de Defensa Legal.

Basombrio C., Rospigliosi F., 2006. La seguridad y sus instituciones en el Peru a inicios del siglo XXI. Reformas democraticas o neomilitarismo. Lima, IEP.

Bazan C., 2013. La clave son las juntas. Ideele, n²29.

Bey M., 1995. La continuité entre villes et campagne au Pérou. Le rôle des associations urbaines. Tiers-Monde, 36 (141), p. 211-222.

Carrillo Florez F., 2007. Seguridad ciudadana en América Latina : un bien publico cada vez mas escaso. Pensamiento Iberaméricano, (0), 181-198.

Costa G., Romero C., 2010. Inseguridad ciudadana en Lima, Que hacer? Lima, Ciudad Nuestra. 
Davila Altamirano D., 2011. Los "ojos y oidos". El rol de las juntas vecinales de seguridad ciydadana en el distrito de San Juan de Lurigancho. Patios de sociales.

Degregori C I., 1996. Cosechando tempestades : Las rondas campesinas y la derrota de Sendero Luminoso en Ayacucho.In Las rondas campesinas y la derrota de Sendero Luminoso, Lima, p. 189-226.

Fuentes Diaz A., 2004. Lynching in Mexico. Ecuador Debate, n61, p. 259-270.

Gervais M., Roussel S., 1998. De la sécurité de l'État à celle de l'individu: l'évolution du concept de sécurité au Canada (1990-1996). Etudes Internationales, 29 (1), p. 25-51.

Goldstein D. M. 2005. Flexible Justice: Neoliberal Violence and "Self-Help" Security in Bolivia. Critique of Anthropology, 25 (4), p. 389-411.

Hine Kelly D., 1997. Vigilantism revisited: an economic analysis of the law of extra-judicial selfhelp or why can't Dick shoot Henry for stealing Jane's truck. Am. UL Rev., n 47, p. 1221.

Huggins M K., 1991. Vigilantism and the state in modern Latin America: essays on extralegal violence. Greenwood Publishing Group.

Johnston L., 1996. What is vigilantism? British Journal of Criminology , n³6 (2), p. 220-236.

Lagos M., Dammert L., 2012. La seguridad ciudadana. El problema principal de América Latina. Lima, Corporacion Latinobarometro.

Lautier B., 2001. Sous la morale, la politique. La Banque Mondiale et la lutte contre la pauvreté. Politique Africaine, 82 (2), p. 169-176.

Lossio Chavez F., 2008. "Ahi si hubo justicia". Linchmientos en el Peru actual. Debates en Sociologia, $\mathrm{n}^{\circ} 33$, p. 117-139.

Pérez Mundaca J., 1996. Rondas campesinas. Poder, violencia y autodefensa en Cajamarca Central. Documento de trabajo, $n^{\circ} 78$, p. 40.

Plöger J. 2006. Practices of socio-spatial control in the marginal neighbourhoods of Lima, Perú. Trialog, 89 (2), p. 32-36.

Pratten D., Sen A., 2008. Global vigilantes: perspectives on justice and violence. In Pratten D. Global vigilantes, edited by David and Atreyee Sen, 1-24. New York: Columbia University Press.

Sierra A., Tadié J., 2008. La ville face à ses marges. Autrepart, 45, p. 3-13.

Tanaka M., 2001. Lima.

Wievorka M., 2005. Penser la violence : en réponse à Sergio Adorno. Cultures et Conflits, 59 (3), p. 175-184.

Yepez Davalos E., 2004. Seguridad ciudadana. 14 lecciones fundamentales. Lima, Instituto de Defensa Legal.

Youngers C., 2003. Violencia politica y sociedad civil en el Peru. Historia de la coordinadora de derechos humanos. Lima, IEP. 


\section{NOTES}

1. Programme Andin de Formation et de Recherche sur les Risques et Vulnérabilités en milieu Urbain, UMR Prodig. L'auteure a fait partie de l'équipe dans le cadre d'un post doc de l'IRD.

2. Instituto de Altos Estudios Policiales.

3. La ville de Lima est divisée en 43 districts qui bénéficient d'une grande autonomie vis-à-vis du pouvoir métropolitain, notamment dans le cadre de la gestion de la sécurité publique via la mise en place de polices municipales. 6 autres districts - augmentés d'un septième en 2014 - font aussi partie de l'agglomération mais dépendent quant à eux de la province du port, le Callao.

4. Que l'on pourrait traduire par « juntes de voisinage de sécurité citoyenne ».

5. Les Comités d'autodéfense sont reconnus par le Decreto Ley n ${ }^{\circ} 741$ du 8 novembre 1991 et leur fonctionnement se voit réglementé par le Decreto Supremo ${ }^{\circ}$ 077/DE-92 DE du 19 Octobre 1992.

6. Entre 1980 et 2000 le Pérou traverse une période d'intense violence politique qui provoque la mort de près de 70000 personnes. Selon les conclusions du rapport final de la CVRN les forces de l’ordre (police plus armée) seraient responsables de près de $30 \%$ des victimes.

7. Avant 1988 la police péruvienne se composait de trois institutions distinctes: la Police d'Investigation (PIP), la Garde Républicaine et la Garde Civile (selon le modèle espagnol).

8. Dirección de Participación Ciudadana.

9. Lima est en croissance constante depuis 1940, en raison notamment de fortes migrations internes par le biais d'invasions de terres qui peu à peu deviennent des quartiers résidentiels, puis des districts, ce qui a pu occasionner des frictions entre l'État et les migrants par rapport à la légitimité de l'occupation du sol.

10. Sources venant des directions de Participation Citoyenne de Lima et Callao, respectivement $7^{\text {ème }}$ et $20^{\text {ème }}$ régions de police selon la juridiction territoriale de la PNP.

11. Local (municipalités de districts et de provinces), régional, national.

12. Les membres de la police nationale péruvienne se divisent en deux corps distincts (euxmêmes structurés par plusieurs grades hiérarchiques) : les officiers et, leurs subordonnées, les sous-officiers. La possibilité de faire carrière dans l'un ou l'autre corps se fait au moment de postuler aux concours d'entrée des écoles de police.

13. Ces chiffres nous ont été fournis par le Bureau de Participation Citoyenne (OPC) de la direction policière régionale pour l'ensemble de Lima Métropolitaine.

14. Les officiers de police péruviens travaillent généralement sur des « gardes » de vingt-quatre heures ; un jour sur deux. Ce qui explique le besoin d'aménagement des infrastructures.

15. Entretien mené par l'auteure le 17 février 2012 à Villa El Salvador.

16. Entretien mené par l'auteure le 09 février 2012 à La Victoria.

17. Plusieurs entretiens réalisés au courant des années 2012 et 2013.

18. Entretien mené par l'auteure le 09 février 2012 à La Victoria.

19. Entretien mené par l'auteure le 29 octobre 2013 à San Juan de Lurigancho.

20. Fumeurs de marihuana et de crack.

21. Entretien mené par auteur le 09 février 2012.

22. Cette anecdote, il la raconte l'enregistreur coupé et alors qu'il nous accompagne au taxi, ses paroles étant couvertes par le flot de la circulation.

23. Chaque municipalité péruvienne est chargée depuis 2003 de former un comité de sécurité citoyenne CODISEC: Comité Distrital de Seguridad Ciudadana) censé réunir le maire, le commissaire (ou représentant de la police nationale), un représentant religieux et un représentant de la société civile (ce qui devrait être logiquement échoir aux dirigeants locaux des JVSC mais cela n'est pas systématiquement le cas).

24. Entretien mené par l'auteure le 12 février 2013. 


\section{RÉSUMÉS}

L'insécurité représente l'une des premières préoccupations des liméniens. Acteur traditionnel du maintien de l'ordre, la police doit aujourd'hui collaborer avec de nouveaux types d'acteurs dont ceux issus du secteur privé ou la population elle-même. C'est ainsi que sont apparus à la fin des années 1990 les Juntas Vecinales de Seguridad Ciudadana, des organisations formées dans le but de seconder la police dans ses tâches de vigilance dans les quartiers les plus défavorisées de Lima. Ces Juntas apparaissent ainsi comme une alternative face au manque de moyens dans les quartiers les plus marginalisés. En même temps, elles disposent de peu de ressources et se trouvent soumises aux diverses rivalités confrontant les différents acteurs de la gouvernance urbaine. Leur champ d'action, tout comme leur légitimité, sont donc relativement limités et elles sont avant tout le reflet des fortes inégalités sociales et spatiales caractérisant la ville de Lima.

\section{INDEX}

Mots-clés : Lima, Juntas Vecinales de Seguridad Ciudadana, vigilantisme urbain, police, participation sociale

\section{AUTEUR}

\section{CAMILLE BOUTRON}

Camille Boutron, boutcam@gmail.com, est sociologue politique, post-doctorante à l'IRD et membre de l'UMR Prodig. 